\title{
METALURGIA CALCOLÍTICA EN CARMONA (SEVILLA)
}

\section{COPPER AGE METALLURGY IN CARMONA (SEVILLA)}

\author{
por
}

SALVADOR ROVIRA ${ }^{1}$

PABLO GÓMEZ RAMOS ${ }^{2}$

RESUMEN El preșente trabajo aborda el estudio científico de minerales, escorias y objetos de cobre calcolíticos recuperados en dos excavaciones realizadas hace pocos años en la ciudad Carmona. El estudio de las escorias es de especial interés dada la escasez de este tipo de material pirometalúrgico en contextos tan antiguos. El análisis con microscopio electrónico de barrido demuestra que se trata de escorias inmaduras, formadas por conglomerados de mineral de cobre y restos de roca encajante, parcialmente fundidos, que remiten a una tecnología primitiva de obtención de cobre que puede ser puesta en relación con los inicios de la metalurgia en otros puntos del Mediterráneo oriental.

ABSTRACT This paper faces the scientific study of Chalcolithic minerals, slags and copper objects recovered few years ago, in two excavations, in the city of Carmona. The study of slags is of particular interest owed to the scarcity of this kind of pyrometallurgical material belonging to so early archaeological contexts. Scanning electron microscope analysis reveals that they are immature slags constituted by a conglomerate of copper minerals and host-rock, partially smelted, which underline a primitive technology that can be related to the beginning of metallurgy in other sites in the Levant.

1. Museo Arqueológico Nacional, Madrid.

2. Becario post-doctoral de la Comunidad de Madrid, Universidad Autónoma de Madrid. 


\section{INTRODUCCIÓN}

Durante las excavaciones realizadas por Elisabet Conlin en la calle Dolores Quintanilla de Carmona se recuperaron varios fragmentos de materiales relacionados con actividades metalúrgicas (escorias y minerales) y tres piezas metálicas. La cronología de estos materiales se encuadra en un Calcolítico pleno sin campaniforme, según la mencionada investigadora.

El conjunto de materiales, si bien modesto en número, resulta de extraordinario interés debido, por un lado, a la parquedad de estudios de escorias calcolíticas realizados hasta ahora utilizando los poderosos recursos de la microscopía electrónica de barrido, y por otro, a la escasez de hallazgos de escorias de estas primeras etapas de la metalurgia del cobre.

A mediados de los 80 descubríamos que, en sus inicios, la obtención de cobre se realizaba de un modo muy sencillo en simples vasijas domésticas que denominamos vasijas-horno (Rovira 1989 y 1998, Rovira y Montero 1994) y que ahora resulta más adecuado llamarlas vasos o vasijas de reducción ${ }^{3}$. Se ha podido constatar que este procedimiento se encuentra muy extendido por la Península Ibérica (Gómez Ramos 1996 y 1999) y, más recientemente, que no es exclusivo de nuestras latitudes sino que también se han encontrado sus rastros en Francia (Carozza 1998).

Un procedimiento de reducción de los minerales de cobre como el propuesto debía partir de menas oxídicas ricas en metal, con poca ganga, lo cual explicaría la producción de pocos residuos escoriáceos. Los análisis de minerales encontrados en los yacimientos avalaban dicha suposición. Pero seguíamos sin conocer con detalle la estructura de las escorias resultantes, cuya analítica y primeros estudios al microscopio óptico apuntaban más bien hacia los conglomerados de horno que hacia las verdaderas escorias (Gómez Ramos 1996 y 1999).

El subsiguiente estudio de laboratorio se ha efectuado empleando la microscopía óptica y la microscopía electrónica de barrido ${ }^{4}$.

\section{LOS MINERALES DE CARMONA}

Se ha analizado un total de tres muestras de minerales de cobre procedentes del Fondo 1 de la calle Dolores Quintanilla. Los resultados analíticos se encuentran en la Tabla 1.

Las muestras CAR9 y CAR 10 corresponden a minerales oxídicos de cobre muy puros, como indican los análisis generales de las mismas ${ }^{5}$. Los contenidos de hierro y de sílice son muy bajos y no se aprecia la presencia de azufre. Todo parece indicar que el fundidor calcolítico seleccionaba los minerales más ricos o trabajaba las vetas más puras de mineral.

La figura 1 muestra el aspecto de una sección pulida del mineral CAR9, en la que se aprecian formaciones cristalinas de color gris claro, probablemente de cuprita.

3. Los días 27 y 28 de febrero de 1999 tuvo lugar en Madrid una reunión de un reducido grupo de investigadores europeos, bajo los auspicios del Instituto Arqueológico Alemán, para discutir aspectos socio-tecnológicos de la metalurgia primitiva. Uno de los temas abordados fue el de las vasijas-horno y su funcionamiento. Recogiendo las acertadas sugerencias de Anthony Hauptmann, resulta más apropiado denominarlas vasos de reducción de mineral de cobre ya que el concepto de horno implica la existencia de una cavidad cerrada y ese no es el caso.

4. Microscopio electrónico del Servicio Interdepartamental de Investigación de la Universidad Autónoma de Madrid, operado por Esperanza Salvador.

5. Los análisis generales se han realizado con la microsonda electrónica, barriendo un campo representativo de la muestra a 50 aumentos. En los demás análisis la técnica ha sido la misma, abriendo pequeñas ventanas sobre el objeto a analizar. Se han evitado en lo posible los análisis puntuales. 
Tabla 1

\begin{tabular}{|l|c|c|c|c|c|c|c|c|c|}
\hline \multicolumn{1}{|c|}{ Objeto } & $\mathrm{O}$ & $\mathrm{Si}$ & $\mathrm{S}$ & $\mathrm{Cl}$ & $\mathrm{Fe}$ & $\mathrm{Cu}$ & $\mathrm{As}$ & Análisis & Inventa \\
\hline Mineral cobre (general) & & 1.7 & 0 & 0 & 1.1 & 96 & 0 & CAR10-1 & DQ-Fon 1 \\
\hline Mineral cobre (calcopirita) & & 0 & 40.48 & 0 & 30.95 & 28.57 & 0 & CAR7-1 & DQ-Fon 1 \\
\hline Mineral cobre (calcopirita) & 0 & 8.82 & 0 & 22.65 & 68.53 & 0 & CAR7-2 & DQ-Fon 1 \\
\hline Mineral cobre (formaciones gris claro) & & 1 & 0 & 0 & 0.2 & 98.3 & 0 & CAR9-1 & DQ-Fon 1 \\
\hline Mineral cobre (general) & 1.5 & 0 & 0 & 3 & 92.4 & 0 & CAR9-2 & DQ-Fon 1 \\
\hline
\end{tabular}

Muy interesante es la muestra CAR7, de calcopirita (sulfuro de cobre y hierro). Es un mineral de color grisáceo tornasolado muy distinto a los verdes o azulados de la malaquita o azurita (carbonatos), o a los pardos rojizos de la cuprita (óxido). En realidad, los sulfuros son los minerales primarios que, por los fenómenos de meteorización, se transforman en los minerales oxídicos secundarios (óxidos y carbonatos). $\mathrm{Su}$ aprovechamiento metalúrgico requiere operaciones más complejas que la simple reducción, pues previamente han de ser tostados a baja temperatura para eliminar el azufre y convertirlos en óxidos. En la Península Ibérica, rica en menas secundarias de cobre cuyos filones no se agotaran durante el Calcolítico y la Edad del Bronce a tenor de la información disponible, no se desarrolló una tecnología adecuada para beneficiar los sulfuros, a diferencia de lo que ocurrió en la Europa Central donde los fahlerz (piritas de Mitterberg y similares) obligaron desde un primer momento a desarrollar métodos de enriquecimiento del mineral y lechos de tostación. No parece, pues, que la calcopirita de Carmona deba interpretarse como signo del beneficio de esta mena de cobre, que raramente ha sido encontrada en yacimientos tan antiguos ${ }^{6}$.

La muestra CAR7 es una calcopirita muy meteorizada a compuestos oxidados, por lo que su proximidad a la veta oxídica que estaban beneficiando los mineros prehistóricos es bastante evidente. La figura 2 muestra una sección vista al microscopio óptico: las áreas de color más claro (análisis CAR7-1) corresponden a calcopirita propiamente dicha, que es la que contiene más azufre $(40,5 \% \mathrm{~S})$. En cambio en los abundantes veteados grises (análisis CAR7-2) la tasa de azufre ha descendido al $8,8 \%$, enriqueciéndose en óxido de cobre $(68,5 \% \mathrm{Cu})$.

\section{LAS ESCORIAS DE CARMONA}

Se han analizado tres muestras de subproductos de la obtención de cobre que, a tenor de los resultados analíticos (Tablas 2 y 3 ), no pueden calificarse de escorias propiamente dichas sino de conglomerados de horno, término que engloba unos materiales pirometalúrgicos de composición muy variable formados por minerales de cobre fundidos y sin fundir, incompletamente reducidos, con reacciones parciales con la ganga y otras impurezas. Cuando faltan las escorias maduras, de tipo fayalítico, son indicio de procesos metalúrgicos sencillos aunque no necesariamente poco eficientes.

Un claro ejemplo de material poco homogéneo es la muestra CAR8 que, predominantemente, tiene la composición CAR8-6 fuertemente silicatada, con abundante sílice libre. Otras regiones de la misma muestra tienen las composición CAR8-5, más rica en cobre y hierro.

Una primera lectura de los análisis globales de estos subproductos permite subrayar su carácter de escorias no fayalíticas: las titulaciones de sílice son variables y bajas (excepto CAR8-6) pero, fundamentalmente,

6. Durante el desarrollo del proyecto Arqueometalurgia de la Península Ibérica se analizaron muestras de calcopirita de La Pijotilla, Almizaraque y Túmulo 3/A-12 de Aldeagordillo (Ávila). Véase Rovira et alii. (1997). 
también lo son los de óxido de hierro, elementos básicos para la formación de fayalita. Además, las pérdidas de cobre son muy elevadas, bien en forma de minerales no reducidos o de pequeñas bolitas de metal atrapado en una matriz demasiado viscosa para permitir una buena separación del cobre formado ${ }^{7}$.

Tabla 2

\begin{tabular}{|l|l|l|l|l|l|l|l|l|l|c|}
\hline \multicolumn{1}{|c|}{ Objeto } & $\mathrm{Al}_{2} \mathrm{O}_{3}$ & $\mathrm{SiO}_{2}$ & $\mathrm{~K}_{2} \mathrm{O}$ & $\mathrm{CaO}$ & $\mathrm{FeO}$ & $\mathrm{CuO}$ & $\mathrm{AS}_{2} \mathrm{O}_{3}$ & \multicolumn{1}{c|}{$\mathrm{SO}$} & $\mathrm{Cl}_{2} \mathrm{O}$ & Análisis \\
\hline Escoria (inclusiones $\mathrm{Cu}_{2} \mathrm{O}$ ) & 0 & 0 & 0 & 0 & 0 & 100 & 0 & 0 & 0 & CAR11-1 \\
\hline Escoria (matriz) & 5.24 & 39.96 & 3 & 2.28 & 5.74 & 43.79 & 0 & 0 & 0 & CAR11-2 \\
\hline Escoria (inclusiones) & 2.65 & 9.57 & 0 & 0 & 0.87 & 72.49 & 0 & 0 & 14.42 & CAR11-3 \\
\hline Escoria (eutéctico, fase clara) & 0 & 10.13 & 0 & 0 & 38.36 & 51.49 & 0 & 0 & 0 & CAR12-5 \\
\hline Escoria (eutéctico, fase negra) & 4.14 & 69.58 & 3.16 & 0 & 8.61 & 14.51 & 0 & 0 & 0 & CAR12-6 \\
\hline Escoria (general) & 3.06 & 15.62 & 0.73 & 0 & 45.31 & 32.25 & 0 & 0 & 3.02 & CAR12-7 \\
\hline Escoria (granos de wustita) & 1.08 & 4.90 & 0 & 0 & 90.85 & 3.18 & 0 & 0 & 0 & CAR8-2 \\
\hline Escoria (vidrio de relleno) & 7.81 & 55.54 & 2.74 & 0 & 13.5 & 9.35 & 10.32 & 0.74 & 0 & CAR8-3 \\
\hline Escoria (general) & 2.86 & 26.27 & 0 & 1.53 & 44.62 & 21.27 & 2.15 & 0 & 1.3 & CAR8-5 \\
\hline Escoria (general) & 2.10 & 70.06 & 0 & 0 & 13.06 & 14.58 & 0 & 0 & 0 & CAR8-6 \\
\hline
\end{tabular}

No se detecta prácticamente azufre, lo cual es una prueba inequívoca de que estos subproductos no provienen de la fundición de minerales sulfurados como la calcopirita.

La figura 3 es una sección de la escoria CAR8 en la que se aprecian claramente grandes granos negros de sílice de perfiles abruptos (no rodados) que no han reaccionado con los materiales que los circundan, y que son la causa de la alta tasa de sílice en el análisis CAR8-6. La figura 4 ofrece una estructura muy distinta en la misma muestra. Está formada por una acumulación de cristales de wustita y magnetita (véase la Tabla 3) insertos en una matriz amorfa de silicato complejo con aluminio, potasio, hierro, cobre y arsénico (análisis CAR8-3). Se distinguen dos bolitas de cobre con altos porcentajes de hierro y arsénico (análisis CAR8-1).

Tabla 3

\begin{tabular}{|l|c|c|c|c|c|c|c|c|}
\hline \multicolumn{1}{|c|}{ Objeto } & $\mathrm{O}$ & $\mathrm{Si}$ & $\mathrm{S}$ & $\mathrm{Cl}$ & $\mathrm{Fe}$ & $\mathrm{Cu}$ & $\mathrm{AS}$ & Análisis \\
\hline Escoria (grano $\mathrm{Cu}$ ) & 0 & 0 & 0 & 0 & 0 & 100 & 0 & CAR12-1 \\
\hline Escoria (cloruro) & 0 & 0 & 0 & 17.7 & 0 & 82.3 & 0 & CAR12-2 \\
\hline Escoria (magnetita) & 24 & 0 & 0 & 0 & 76 & 0 & 0 & CAR12-4 \\
\hline Escoria (inclusión blanca) & 0 & 20.99 & 0.46 & 0 & 5.4 & 57.28 & 0 & CAR12-3 \\
\hline Escoria (wustita) & 21 & 0 & 0 & 0 & 79 & 0 & 0 & CAR8-2 \\
\hline Escoria (grano Cu) & 0 & 0 & 0 & 0 & 4.51 & 85.79 & 9.71 & CAR8-1 \\
\hline Escoria (magnetita) & 25 & 0 & 0 & 0 & 75 & 0 & 0 & CAR8-4 \\
\hline
\end{tabular}

7. En la fusión escorificante lo que se persigue con la adición de fundentes es conseguir una escoria de bajo punto de fusión, poco viscosa a la temperatura de trabajo del horno, que permite la separación por diferencia de densidades del cobre que se va formando conforme el mineral fundido es reducido. 
Esta región de la escoria es altamente informativa. Por un lado nos dice que se estaban procesando minerales oxídicos de cobre con arsénico (asociación natural malaquita-olivenita, por ejemplo) a partir de los cuales, de una forma no intencionada, se obtenía cobre arsenical. La composición del metal, con 4,5\% Fe (análisis CAR8-1), indica una temperatura de trabajo algo superior a $\operatorname{los} 1.100^{\circ} \mathrm{C}$, a la cual la solubilidad del hierro: en el cobre fundido es precisamente del orden del $4 \%$. A dicha temperatura los óxidos de hierro (wustita y magnetita) están en estado sólido, incrementando la viscosidad de la matriz pastosa de óxido de cobre fundido. La abundante magnetita, por su parte, es consecuencia de un exceso de flujo de oxígeno que, a alta temperatura, oxida el óxido ferroso ( $\mathrm{FeO}$, wustita) a ferroso-férrico $\left(\mathrm{Fe}_{3} \mathrm{O}_{4}\right.$, magnetita). Este exceso de ventilación parece necesario para mantener el carbón incandescente a alta temperatura en un recipiente tan abierto como suele ser la vasija de reducción. En otras palabras, para alcanzar y mantener una temperatura en la que las reacciones químicas necesarias para transformar el mineral en cobre metálico en un tiempo y con un rendimiento razonables, empleando el vaso de reducción, se requiere un aporte de aire que hace que las condiciones ambientales en el vaso sean oxidantes. Afortunadamente, la presión de monóxido de carbono necesaria para que tenga lugar la reducción es muy baja ${ }^{8}$ y la reacción ocurre aunque las condiciones no sean las ideales. Es muy posible que el flujo alternante de un fuelle o, más probablemente, de unos tubos de soplado a pulmón proporcionen los períodos suficientes de ambiente reductor para que la reacción se produzca.

La muestra CAR11 es otro conglomerado de horno cuya matriz fundida es un silicato complejo en el que se ha medido hasta un $43,7 \%$ de cobre en forma de oxido. Las figuras 5 y 6 permiten observar una estructura heterogénea con granos de sílice libre y formaciones de cuprita.

Finalmente, la escoria CAR 12 es un subproducto de la metalurgia primaria rico en hierro, cobre y silicịo (análisis CAR12-7). Tanto la observación al SEM (fig. 7) como al microscopio óptico (fig. 8́) permiten apreciar la abundancia de formaciones cristalinas de magnetita en cuyos intersticios se han desarrollado estructuras laminares de carácter eutéctico (análisis CAR12-5 y CAR12-6). Hay algunas formaciones de cobre metálico muy puro (análisis CAR12-1). También se aprecia ataque de cloruros en zonas periféricas ricas en cobre (análisis CAR12-2).

\section{LOS METALES DEL ENTORNO DE CARMONA}

Las excavaciones de la calle Dolores Quintanilla proporcionaron también en el Fondo 1 dos objetos metálicos: un punzón fragmentado y una curiosa laminilla de sección rectangular, con un extremo doblado y ligeramente afilado. Los análisis de la composición metálica (Tabla 4) indican que se trata de cobre ligeramente arsenicado.

La posibilidad de obtener cobre con arsénico está avalada por la presencia de este segundo elemento en alguna de las cargas de mineral empleado, como demuestra la escoria CAR8, cuyas inclusiones metálicas contienen casi un $10 \%$ As.

Aunque el número de objetos recuperados es pequeño para usarlo en pruebas estadísticas, sus composiciones resultan bastante coherentes si las comparamos con los de materiales de El Acebuchal, yacimiento del Bronce Antiguo del término de Carmona. Dejando aparte la cuestión del arsénico (muy variable en estos casos), ambos grupos de metales se caracterizan por contener pocas impurezas de plata y de antimonio, lo que en cierto modo remite a un modelo de aprovechamiento de recursos metalíferos con cierta similitud, a pesar del hiato temporal que posiblemente los separa.

8. Esta es la razón principal por la cual el cobre fue el primer metal obtenido a partir de sus menas. 
Tabla 4.

Metales antiguos de Carmona (\% en peso)

\begin{tabular}{|l|c|c|c|c|c|c|c|c|c|c|c|}
\hline \multicolumn{1}{|c|}{ Objeto } & $\mathrm{Cu}$ & $\mathrm{As}$ & $\mathrm{Sn}$ & $\mathrm{Pb}$ & $\mathrm{Fe}$ & $\mathrm{Ni}$ & $\mathrm{Zn}$ & $\mathrm{Ag}$ & $\mathrm{Sb}$ & Análisis & Yacimiento \\
\hline Pieza laminar & 99.87 & 0.10 & $\mathrm{Nd}$ & $\mathrm{Nd}$ & $\mathrm{Nd}$ & $\mathrm{Nd}$ & $\mathrm{Nd}$ & $\mathrm{Nd}$ & 0.02 & $\mathrm{DQ6-1}$ & c/ Dolores \\
\hline Punzón (frag.) & 99.68 & 0.30 & $\mathrm{Nd}$ & $\mathrm{Nd}$ & $\mathrm{Nd}$ & $\mathrm{Nd}$ & $\mathrm{Nd}$ & $\mathrm{Nd}$ & 0.01 & DQ6-2 & c/ Dolores \\
\hline Punta Palmela & 98.95 & 0.70 & $\mathrm{Nd}$ & 0.09 & 0.24 & $\mathrm{Nd}$ & $\mathrm{Nd}$ & 0.002 & 0.006 & PA6563 & El Acebuchal \\
\hline Punta Palmela & 99.10 & 0.69 & $\mathrm{Nd}$ & $\mathrm{Nd}$ & 0.21 & $\mathrm{Nd}$ & $\mathrm{Nd}$ & $\mathrm{Nd}$ & $\mathrm{Nd}$ & PA6572 & El Acebuchal \\
\hline Punta Palmela & 99.34 & 0.50 & $\mathrm{Nd}$ & $\mathrm{Nd}$ & 0.14 & $\mathrm{Nd}$ & $\mathrm{Nd}$ & 0.016 & 0.007 & PA6566 & El Acebuchal \\
\hline Punta pedunculada & 97.81 & 2.1 & $\mathrm{Nd}$ & $\mathrm{Nd}$ & 0.05 & $\mathrm{Nd}$ & $\mathrm{Nd}$ & 0.006 & 0.020 & PA6569 & El Acebuchal \\
\hline Punta Palmela & 99.06 & 0.66 & 0.024 & 0.07 & 0.10 & 0.07 & $\mathrm{Nd}$ & $\mathrm{Nd}$ & 0.003 & PA6570 & El Acebuchal \\
\hline Punta Palmela & 99.21 & 0.57 & 0.062 & 0.08 & 0.07 & $\mathrm{Nd}$ & $\mathrm{Nd}$ & 0.005 & 0.004 & PA6571 & El Acebuchal \\
\hline Puñal lengüeta & 97.07 & 2.5 & $\mathrm{Nd}$ & $\mathrm{Nd}$ & 0.44 & $\mathrm{Nd}$ & $\mathrm{Nd}$ & 0.003 & 0.010 & PA6561 & El Acebuchal \\
\hline Punta Palmela & 98.93 & 0.37 & $\mathrm{Nd}$ & 0.13 & 0.24 & $\mathrm{Nd}$ & $\mathrm{Nd}$ & 0.015 & 0.014 & PA6573 & El Acebuchal \\
\hline
\end{tabular}

(Los análisis de El Acebuchal han sido tomados de Rovira et alii. 1997)

\section{CONSIDERACIONES FINALES}

El reciente estudio de las técnicas de obtención de metal en la Península Ibérica (Gómez Ramos 1999: 75-77) traza con cierto detalle los rasgos de la tecnología calcolítica. Ahora estamos en condiciones de aportar nuevos datos técnicos que avalan algunos de dichos rasgos. Las escorias de la calle Dolores Quintanilla, inmaduras, encajan bien como subproducto escaso de los vasos de reducción empleados para la producción de cobre. Sabíamos por los estudios microscópicos de las escorificaciones de estos vasos y de otras escorias que las condiciones en las que se producía la transformación del mineral en metal eran más bien oxidantes que reductoras. Áhora podemos confirmar que es así, por la abundancia de magnetita en las escorias.

Se confirma también que no hay intencionalidad en la adición de fundentes para formar verdaderas escorias, y que la presencia de sílice libre y altas tasas de hierro se debe, por un lado, al acompañamiento de roca encajante junto al mineral machacado y a la propia complejidad de la mena, y por otro, a la concentración residual de ganga a lo largo del proceso reductor.

De la naturaleza de la escoria se deduce que los minerales beneficiados proceden de una mineralización encajada en cuarzo, dato a tener en cuenta a la hora de buscar su posible origen.

Estas escorias y el procedimiento de obtención de cobre que las produce puede ponerse en relación tecnológica con las primeras producciones jordanas de Wadi Fidan 4, fechadas en el IV milenio a.C. (Calcolítico final), estudiadas por Hauptmann et alii. (1996). Aunque entre ambas regiones hay ciertas variantes de procedimiento ${ }^{9}$, substancialmente remiten a un mismo objetivo: obtener cobre directamente de los minerales usando vasos de reducción, sin necesidad de verdaderos hornos metalúrgicos. En Jordania este fue un estadio tecnológico inicial que luego sería sustituido por las fundiciones escorificantes en los hornos que caracterizan la metalurgia de Feinan del Bronce Antiguo y de épocas posteriores (Hauptmann, com. personal). En nuestras latitudes la evolución tecnológica discurrió por otros derroteros, pero conviene señalar que en sus comienzos se atuvo a un patrón como el de Jordania y otros puntos del Levante mediterráneo donde se explotaban especies minerales como las españolas. Esta tecnología, ligada más a las leyes generales fisicoquímicas

9. En Wadi Fidan se usaban crisoles enmangados, de entre 9 y $11 \mathrm{~cm}$ de diámetro. 
aplicadas a los recursos disponibles que a los condicionamientos culturales, es un indicio más para avalar la propuesta del descubrimiento autóctono de la metalurgia en la Península.

(Agradecimientos: Agradecemos a Elisabet Conlin el habernos proporcionado los materiales para este estudio y a María Belén Deamos el soporte financiero necesario para costear los análisis a través de sus proyectos de investigación).

\section{BIBLIOGRAFÍA}

CAROZZA, L. 1998: “Analyse et interprétation des témoins de métallurgie Chalcolithique découverts dans l'habitat d'Al Claus (Tarn-et-Garonne)". En M.-Ch. FRÈRE-SAUTOT (ed.): Paléométallurgie des Cuivres. Éditions Monique Mergoil. Montagnac: 45-51.

GÓMEZ RAMOS, P. 1996: "Hornos de reducción de cobre y bronce en la Pre y Protohistoria de la Península Ibérica”. Trabajos de Prehistoria 53 (1): 127-143.

1999: Obtención de metales en la Prehistoria de la Península Ibérica. BAR International Series 753. Oxford.

HAUPTMANN, A., BACHMANN, H.-G., MADDIN, R. 1996: "Chalcolithic copper smelting: new evidence from excavations at Feinan, Jordan". Archaeometry 94. The Proceedings of the 29 International Symposium on Archaeometry. Ankara 9-14 May 1994. TÜBITAK. Ankara: 3-10.

ROVIRA, S. 1989: "Recientes aportaciones para el conocimiento de la metalurgia primitiva en la provincia de Madrid: un yacimiento Campaniforme en Perales del Río(Getafe, Madrid)". Actas del XIX Congreso Nacional de Arqueología. Castellón, 1987. Vol. I. Zaragoza: 355-366.

1998: "Metalurgia campaniforme en España: resultados de quince años de investigación arqueometalúrgica". En M.-Ch. FRËRE-SAUTOT (ed.): Paléométallurgie des Cuivres. Éditions Monique Mergoil. Montagnac: 109-127.

ROVIRA, S., MONTERO, I. 1994: "Metalurgia Campaniforme y de la Edad del Bronce en la Comunidad de Madrid”. En C. BLASCO (ed.): El Horizonte Campaniforme de la Región de Madrid en el Centenario de Ciempozuelos. Univ. Autónoma de Madrid. Madrid: 137-171.

ROVIRA, S., MONTERO, I., CONSUEGRA, S. 1997: Las Primeras Etapas Metalúrgicas en la Península Ibérica. I. Análisis de Materiales. Instituto Universitario Ortega y Gasset y Ministerio de Educación y Cultura. Madrid. 


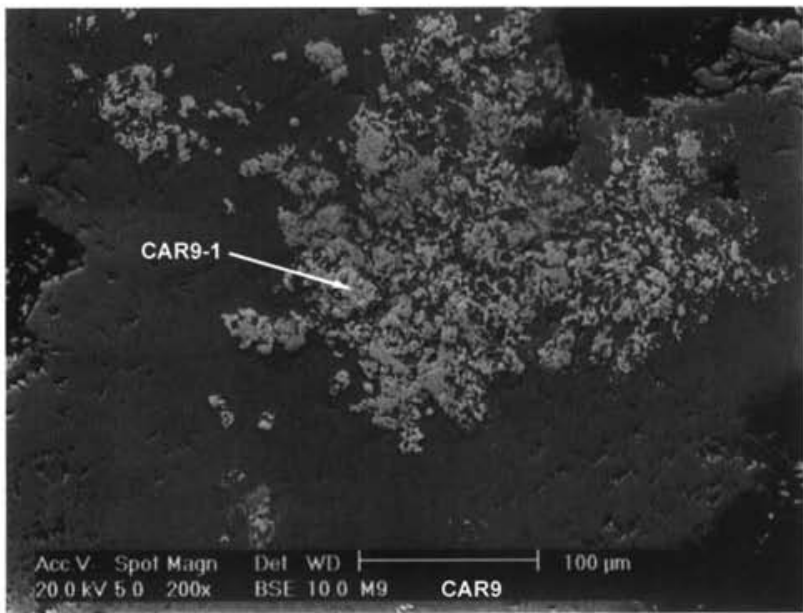

Fig. 1. Imagen SEM con electrones retrodispersados del mineral de cobre CAR9. Las formaciones de color gris claro son cristalizaciones, probablemente cuprita.

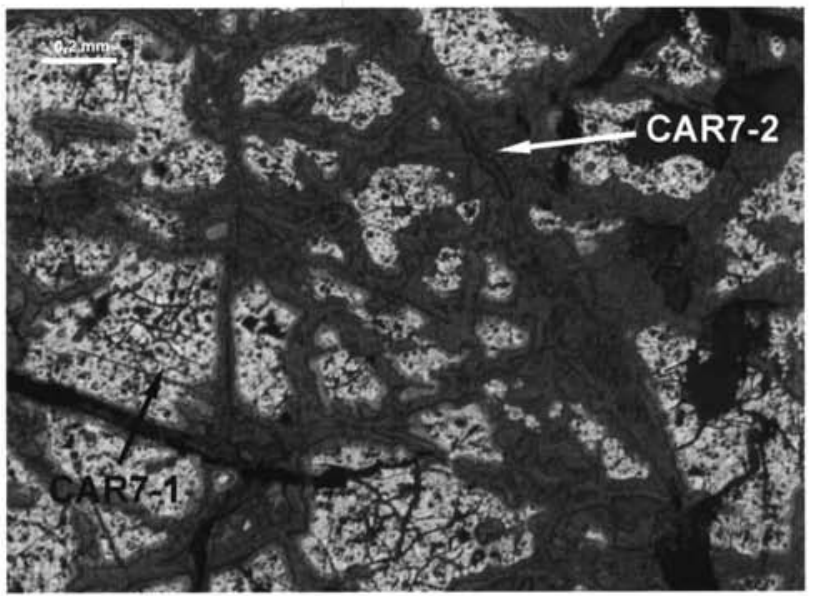

Fig. 2. Imagen al microscopio óptico (iluminación de campo claro) de la calcopirita CAR7. Obsérvese el veteado gris de la fase meteorizada. 


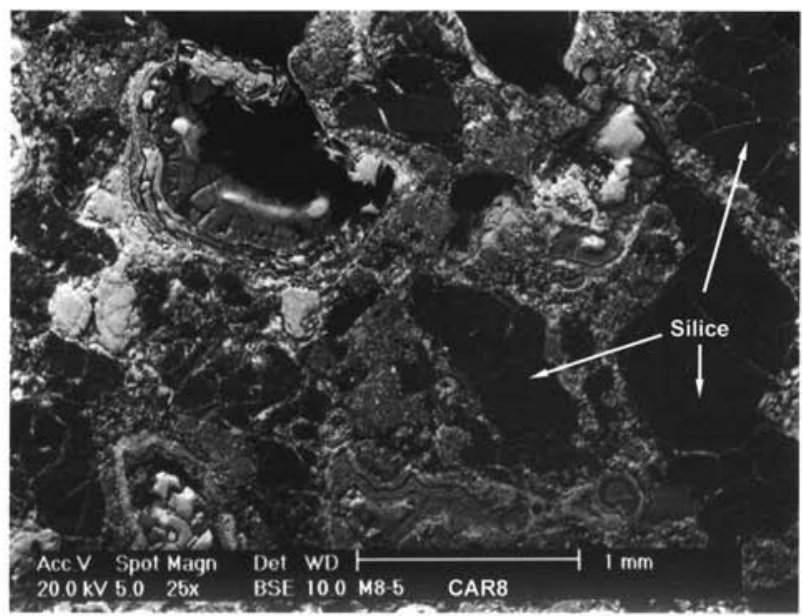

Fig. 3. Imagen SEM con electrones retrodispersados de la muestra CAR8. Obsérvese la abundancia de granos de silice libre.

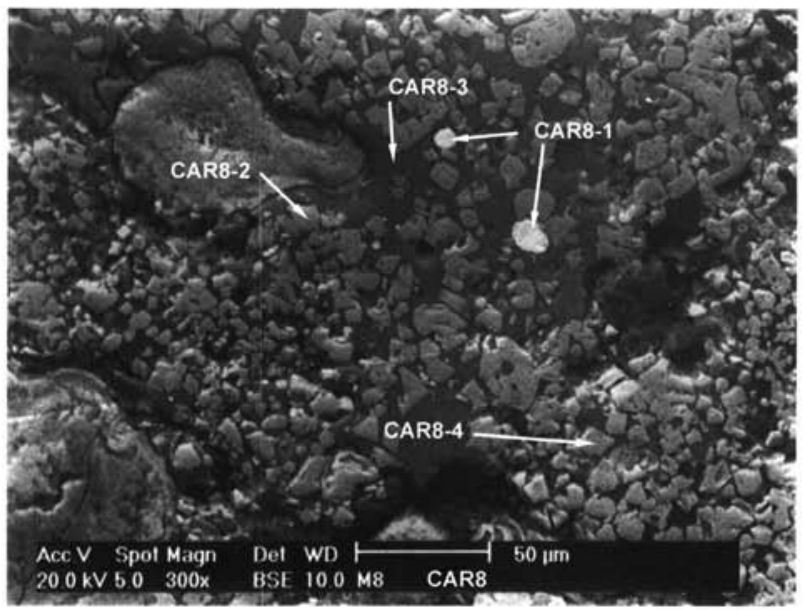

Fig. 4. Imagen SEM con electrones retrodispersados de una región de la muestra CAR8. Glóbulos y cristales de óxidos de hierro tapizan el campo, en el que se aprecian también dos pequeñas esferas de cobre metálico (color blanco). 


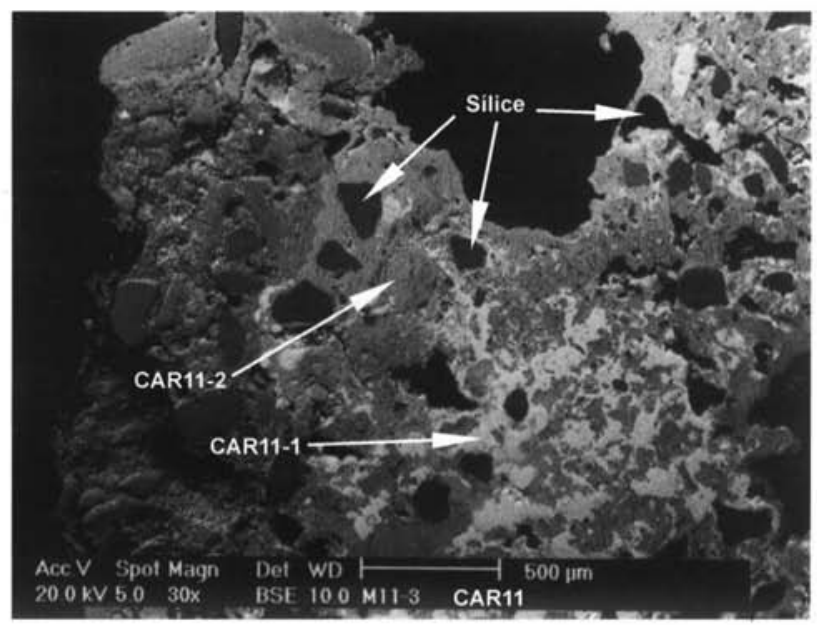

Fig. 5. Imagen SEM con electrones retrodispersados de la muestra CAR11. Se aprecian granos negros de silice libre y formaciones de cuprita blanca en una matriz de silicato complejo con abundante cobre.

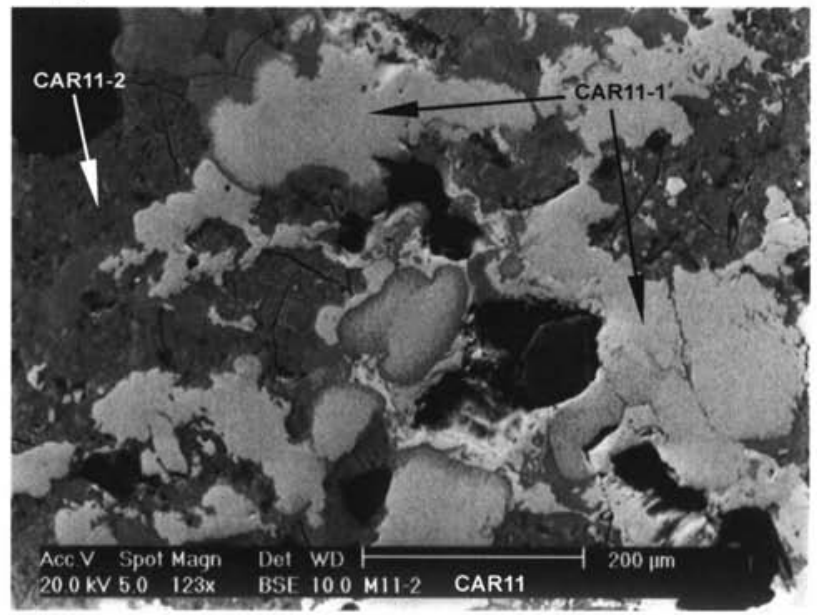

Fig. 6. Imagen SEM con electrones retrodispersados de la muestra CAR11. Detalle de las formaciones de cuprita. 


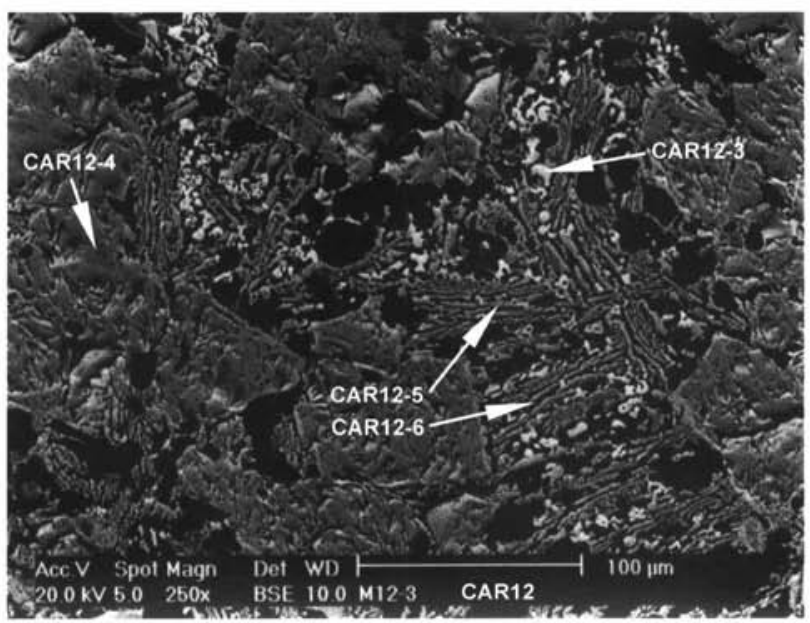

Fig. 7. Imagen SEM con electrones retrodispersados de la muestra CAR12. Detalle de las formaciones laminares eutécticas entre granos de magnetita.

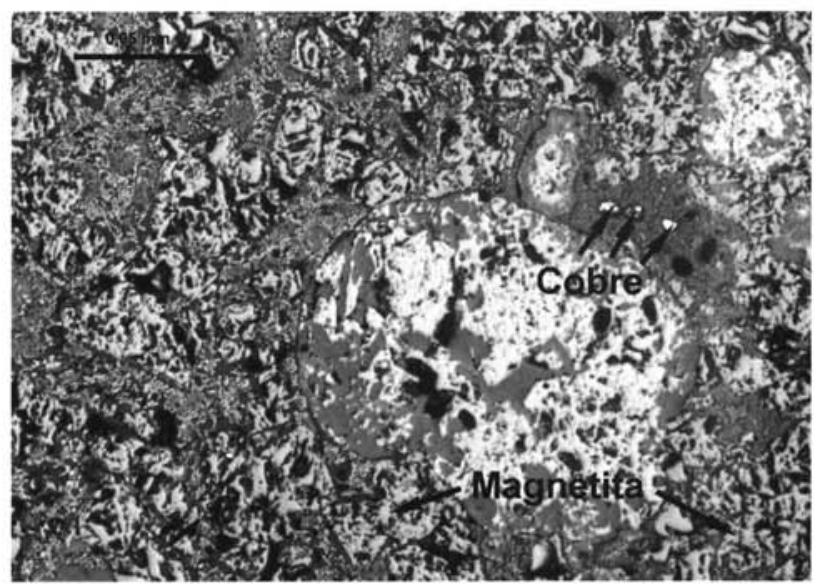

Fig. 8. Imagen del microscopio óptico en la que se aprecian tres granitos de cobre metálico. 